Quaderni di Geografia Cahiers de Gécongaty rataphie

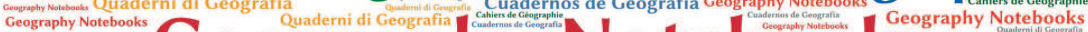
Cuadernos de Geografia Geography Notebooks $U 201000$ Cahiers de Géographi Cahiers de Géographie cahiers de Géographie Cuadernos de Geografía Cahiers de Géographie Cahiers de Géographie Cuadernos de Geografía Geography Notebooks

\author{
$4(2021)$ \\ 1 \\ Teatro di suoni. \\ Spazi acustici teatrali e territoriali
}

A cura di

Martino Mocchi, Lorena Rocca, Demis Quadri and Carlotta Sillano

EDITORIAL

Teatro di suoni per l'attaccamento ai luoghi. Uno sguardo geografico 11

Lorena Rocca

Per un teatro di suoni. Riflessioni su possibili dimensioni sonore nelle 23 creazioni site-specific di physical theatre

Demis Quadri

INTRODUCTION

Teatro di suoni. Spazi acustici teatrali e territoriali

Demis Quadri e Lorena Rocca

SPECIAL Issue

I suoni di Mantova come strumenti di interpretazione del paesaggio.

Tra turismo sostenibile ed educazione al patrimonio culturale

Valeria Pecorelli, Franca Zuccoli, Alessandra De Nicola, Enrico Squarcina

Il paesaggio sonoro campano tra contemporaneità e nuove forme

di progettualità turistica

Germana Citarella 
La narrazione spettacolarizzata del paesaggio sonoro.

Da Giuseppe Chiari a Philip K. Dick e oltre

Francesco Michi

Musica di paesaggi sonori. Enunciazione, risignificazione, comunicazione

Carlotta Sillano

Camminare per ascoltare. Partiture invisibili del territorio abitato

Elisabetta Senesi

Il paesaggio sonoro in relazione. Suono, movimento e immagini per stimolare complessità percettiva Angela Calia

Groove Fields. Understanding the Dance Floor from an Art-Based Research Perspective

Sebastian Mattbias

Il silenzio come esperienza trasformativa. L'importanza del silenzio nella meditazione e in ambito professionale

Sebastiano Caroni

Progettare il silenzio. Una lettura acustica dell'ex villaggio sanatoriale 125 Morelli a Sondalo

Martino Mocchi

Voicing One's Will. Theatre as Audio-Visual Hypotyposis of the Poetic

Michael Groneberg

Music and Clowning in Europe, 20th-21st centuries

Anna Stoll Knecht

Il paesaggio sonoro nella composizione musicale. Un percorso bibliografico

Stefano Alessandretti

\#exploreART: il labirinto di A. Pomodoro e i bambini. Un progetto di fruizione condivisa con percorsi sensoriali partecipati Alessandra De Nicola, Franca Zuccoli 


\section{OTHER EXPLORATIONS}

Il rumore lontano. Intervista a Lorena Rocca

a cura di Martino Mocchi

Re Cervo. Intervista a Antonella Astolfi

a cura di Krizia Bonaudo e Demis Quadri

Centovalli-Centoricordi. Intervista a Oliviero Giovannoni a cura di Krizia Bonaudo e Demis Quadri

Alla ricerca di un metodo: Open Space Technology 



\title{
Musica di paesaggi sonori. Enunciazione, risignificazione, comunicazione
}

\author{
Carlotta Sillano \\ Dipartimento DFA, SUPSI \\ DOI: https://doi.org/10.7358/gn-2021-001-sill
}

\begin{abstract}
The paper - rapidly retracing some fundamental stages in the history of sound studies - questions the relationship between soundscape and music language and proposes a reflection on the resulting ontologies of sound. In the traditional conception of soundscape studies, musical language should contribute to a re-orchestration of environmental sound and should promote awareness and active listening of extra-musical sounds. However, every music composition or performance that draws on this kind of acoustic material, inevitably transforms its fruition. Immediacy is in fact impossible and each perception produces a new meaning. The article highlights the value of subjective perception and creative processes in the multidisciplinary discussion about soundscape.
\end{abstract}

Keywords: soundscape; music; sound ontology; music semiology; acoustic communication.

Parole chiave: paesaggio sonoro; musica; ontologia del suono; semiotica musicale; teorie della comunicazione.

Io lavoro, mi diceva all'orecchio, a cose totalmente futili.

La parola velluto per esempio, non è come sentire del velluto?

Un che di morbido, di peloso 
sulla lingua.

Si può dire?

Si chiama così? - Così cosa?

Questo soffio lanuginoso nel Suo orecchio.

Nel mio orecchio? - Lei rabbrividisce.

E' dunque a questo che Lei lavora! - Sì.

Se si nomina qualcosa, esiste,

si rigonfia,

morbido e senza punte,

pura futilità,

come questo brivido

che è una parola

e non è velluto.

Hans Magnus Enzensberger, Il mentitore ${ }^{1}$

\section{Il SUONO POLITICO, IL SUONO ESTEtico}

La prima formulazione teorica del concetto di paesaggio sonoro si trova, come è noto, nell'affascinante quanto controverso testo di Raymond Murray Schafer The tuning of the world, in cui lo stesso è definito sinteticamente come «l'ambiente dei suoni. Tecnicamente, qualsiasi parte dell'ambiente dei suoni considerata come campo di studio e ricercas (Schafer 1977).

Il testo di Schafer è considerato un documento fondamentale per tutti quegli studi che investigano il rapporto tra suono e spazio, ma anche la relazione tra suono e produzione sociale di significato - ovvero le varie branche dei sound studies e dei soundscape studies. Il testo introduce una teoria dei paesaggi sonori, ma non si tratta di un testo propedeutico e neutrale. Se la definizione di paesaggio sonoro qui riportata appare democratica e aperta a qualsivoglia ambiente acustico, l'autore mira a effettuare una selezione carica di valori insieme estetici e politici.

Quello posto dall'autore non è un invito a trovare una relazione armonica con il mondo, ma a intervenire sul suo suono (e conseguentemente sulla nostra sensibilità nei confronti di esso) per rinvenire una "significativa cultura uditiva". L'obiettivo è limitare, attraverso la sensibilizzazione all'ascolto e alle tecniche del sound design, i suoni nocivi,

\footnotetext{
${ }^{1}$ Enzensberger, H.M. 1997. Musica del futuro, Torino: Einaudi.
} 
per fare ritorno a una sorta di Eden aurale, selezionando o costruendo nostalgicamente mondi sonori "ben temperati".

La prospettiva adottata è dichiaratamente ecologica. L'Ecologia Acustica - la disciplina concepita da Schafer per lo studio della "relazione di equilibrio tra i suoni e il loro ambiente" - non promuove, infatti, esclusivamente una ricerca riguardante la conoscenza della natura del suono e della sua esperienza in un determinato ambiente, ma è volta a innescare azioni pratiche con l'intento di preservare, trasformare, migliorare un dato spazio acustico. La Bibbia del paesaggio sonoro, The Tuning of the World, ristampato nel 1999 con il titolo The Soundscape e tradotto in Italia come Il paesaggio sonoro risulta quindi «un testo prescrittivo cui ci si riferisce spesso come un testo descrittivo» (Kelman 2010).

Schafer intende coinvolgere in questa sua battaglia figure professionali diverse: ricercatori, urbanisti, artisti. Proprio a questi ultimi rivolge un invito diretto ad arruolarsi nell'esercito silenzioso dell'Ecologia Acustica. I responsabili dell'opera di sensibilizzazione voluta da Schafer dovrebbero essere in primis i compositori, chiamati a investire in un'arte sociale:

I compositori, estraniatisi per troppo tempo dalla società, devono oggi tornare a prestare aiuto alla navigazione degli uomini. [...] Non sono però pronti ad assumersi questo ruolo guida nella riorchestrazione dell'ambiente sonoro. Alcuni di loro continuano a dedicarsi, con stizzosa rigidezza, a un Parnaso che non interessa a più di due o tre persone. (1977)

Non c'è dunque spazio per l'art pour l'art nella teoria di Schafer e nemmeno per il suono per il suono, che deve invece essere sempre in contatto con il suo referente, richiamare la sua sorgente, lo spazio in cui riecheggia e i suoi abitanti umani, animali, architettonici, tecnologici. Udire - afferma Schafer - è toccare a distanza; la sua concezione del suono è pragmatica (Serra, 2005).

Questa ideologia include una valutazione estetica dei suoni del mondo; Schafer parla di forma e bellezza, di volgarità e aberrazione. Il suono non è pronto a dirsi da sé, deve essere controllato e riorganizzato per prendere forma e armonia, per essere ascoltato come una composizione musicale: «la questione di fondo è: il soundscape del mondo è una composizione indeterminata su cui non abbiamo alcun controllo, o siamo noi i suoi compositori ed esecutori, responsabili di darvi forma e bellezza?» (Ibid.). 


\section{IL SUONO PER IL SUONO}

Il linguaggio musicale ha intersecato i percorsi del soundscape ben prima che questo fosse teorizzato come tale negli anni Settanta da Schafer e dal suo gruppo di ricerca. I suoni extra-musicali sono stati dapprima evocati, attraverso processi simbolici e imitativi, talvolta più allegorici (il mormorio di un ruscello nella Pastorale di Beethoven, le figure retoriche della tradizione madrigalistica), altre più realistici (l'intonarumori di Russolo).

Grazie agli strumenti di fonofissazione sono poi diventati timbri a diretta disposizione del compositore, in un graduale processo di musicalizzazione del rumore che ha investito il Novecento. Un capitolo fondamentale del percorso frastagliato e controverso che lega il paesaggio sonoro ai linguaggi della musica è rappresentato dall'esperienza di Pierre Schaeffer e della musica concreta. Schaeffer intende sfruttare gli strumenti tecnologici per comporre a partire dal suono, rinunciando alla notazione musicale. Il punto di partenza per la scrittura è il lavoro sulla materia sonora in un processo che esclude il codice visivo (la partitura). Un simile approccio può nascere esclusivamente da un ascolto attivo delle variabili del suono, un ascolto ridotto: «l'ascolto ridotto è l'attitudine di ascolto che consiste nell'ascoltare il suono per se stesso, inteso come oggetto sonoro, facendo astrazione dalla sua provenienza, reale o supposta, e dal senso di cui può essere portatore» (Chion 2004).

L'esperienza acusmatica intende fare del suono il fine ultimo dell'esperienza dell'ascolto: il suono non è una proprietà degli oggetti, ma diviene oggetto a sé stante. Un oggetto monosensoriale, puro nella sua forma acustica. L'acusma, l'oggetto sonoro, costringe a un approccio nuovo rispetto alla materia udita, un ascolto che prevede l'astrazione dal mondo e dai suoi meccanismi e si concentra sulle caratteristiche del materiale musicale. Si tratta di un'epoché; di un decondizionamento dalle abitudini d'ascolto. Il principio non è molto diverso da quello che guida la riduzione fenomenologica teorizzata da Husserl: abbandonare l'atteggiamento naturale rispetto alle cose del mondo per "guardare alle cose stesse" (1965).

Spezzati i vincoli con le sorgenti sonore, la musica concreta è libera di inglobare ogni suono nel discorso musicale, senza distinzione tra suoni d'ambiente, voci, rumori, strumenti musicali. L'oggetto sonoro però non è dato. Ogni suono si trova per sua natura all'interno di un campo sonoro più ampio, in relazione continua con altri suoni e altri stimoli, l'oggetto sonoro deve essere isolato da quello che potremmo chiamare un paesaggio sonoro, l'ambiente dei suoni, e presentato come oggetto 
intenzionale. Deve essere estrapolato e restituito come elemento discreto. In questo, prende nuova forma, una forma che non è in relazione con il mondo, ma è in sola relazione con l'ascoltatore, invitato a trovare un mondo all'interno del suono.

La visione di Schaeffer è spesso stata osservata come antitetica rispetto alla proposta di Schafer - e la somiglianza del suono dei loro nomi pare quasi uno scherzo del destino. Il sound artist Francisco Lopez, che esplora nelle sue opere la connessione tra ascolto acusmatico e field recording, sottolinea come il desiderio di rendere esplicito il legame tra suono e sorgente sia utile a un obiettivo pragmatico ed extra-musicale, ma non all'astrazione propria del linguaggio della musica:

Ci può essere solamente una ragione documentaria o comunicativa per mantenere la relazione causa-oggetto nel lavorare con i paesaggi sonori, non di certo una motivazione artistico-musicale. Sono convinto che più questa relazione sia mantenuta, meno musicale sarà il lavoro. In una composizione musicale (non importa se basata sul paesaggio sonoro oppure no) ci deve essere un'azione libera: non si può dover rinunciare a estrarre gli elementi dalla realtà e si deve avere il pieno diritto di essere auto-referenziali, non essendo soggetti a un obiettivo pragmatico. (2010)

Il programma della musica concreta era di natura sperimentale e come tale era da ritenersi un atto dall'esito incerto, il cui valore si ritrova nella ricerca e non nella riuscita o nell'insuccesso. Le dichiarazioni di Lopez dimostrano come le riflessioni maturate in questo contesto abbiano influenzato e si siano radicate nel pensiero compositivo contemporaneo. Nonostante ciò, alla fine della sua carriera, Pierre Schaeffer ha dichiarato che la sua ideologia è stata un fallimento. L'esperienza della musica concreta aveva creato un modello capace di generare strutture sonore, ma non musica. Schaeffer inizia la sua opera cercando di negare il processo compositivo della musica astratta e finisce per considerarlo l'unico possibile. Risulta di particolare interesse questo passaggio di Grubbs:

Si potrebbe pensare che quando Schaeffer coniò l'espressione "musique concréte" avesse dato per scontato che il mondo dei suoni potesse essere percepito esso stesso come musicale, e che i suoni concreti non richiedessero un sistema particolare perché li si inserisse nel regno della musica. (Grubbs 2014) 
Anche per Schaeffer la percezione estetica dei suoni extra-musicali necessita dunque non solamente di un condizionamento (l'oggettificazione del suono, di per sé un processo di manipolazione) ma di un atto compositivo. L'oggetto sonoro è un pre-testo (Bonnet 2016), che deve essere inserito in un sistema di ordine e relazioni, di ripetizioni, similitudini e differenze.

\section{Dire il PAEsaggio SONORO}

Sono state fin qui molto rapidamente esposte due modalità antitetiche di considerare il suono extra-musicale, oggetto di un processo di musicalizzazione, nel suo rapporto con il mondo. Queste posizioni sono solo due delle molte possibili, ma sono considerate entrambe seminali e fondamentali nella riflessione sull'ontologia del suono extra-musicale in relazione al linguaggio musicale stesso. In entrambe queste ideologie sembra essere comune una necessità di guardare al suono come a uno stimolo che fatica a dirsi da solo, che non può attivare una percezione estetica quando lasciato incontrollato e libero di fluire.

Pare allora che per risultare significativa, la langue inascoltata dei suoni del mondo debba diventare parole, prendere forma e accadere in quello che possiamo paragonare a un atto di enunciazione. Nel compiere questo atto linguistico, ogni suono extra-musicale acquisisce necessariamente un significato nuovo.

Un esempio radicale di quanto affermato si trova nella celebre composizione 4'33" di John Cage. Il compositore intende in modo programmatico farsi da parte e rivolgere un invito all'ascolto dei suoni ambientali, casuali e non ordinati che circondano il pubblico della sua opera, in quello che a tutti gli effetti si configura come un teatro di suoni. Nell'attribuire una durata al suo silenzio, che non è assoluto, ma si configura come una pausa, un tacere (tacet è la sola indicazione in partitura), procura uno slittamento di senso.

Augusto Ponzio traccia una distinzione tra il tacere e il silenzio, derivata dagli Appunti del 1970-71 di Bachtin e dalle lezioni al Collège de France di Barthes:

Il silenzio permette l'identificazione degli elementi reiterabili del discorso, cioè degli elementi che fanno parte della lingua. Il tacere è invece condizione di comprensione del senso della singola enunciazione nella sua irripetibilità. Il silenzio è collegato con entità fisiche, i suoni, 
e con unità astratte della lingua come sistema: fonemi, morfemi, proposizioni, frasi. Il tacere, invece, riguarda l'unità concreta della comunicazione verbale, l'enunciazione nella sua parte non iterabile. (2006)

In questo enunciato silenzioso, che diventa posizione di ascolto, è presente una vocazione, un processo di significazione, quella che per Lévinas è "significanza della significazione"; l'eccedenza del dire rispetto al detto. Il dire non è al servizio del messaggio, ma vale per se stesso e instaura un rapporto. Quella di Cage in quest'ottica è un'invocazione, una forza illocutoria, un richiamo rivolto all'ascoltatore che diventa improvvisamente presente, pronto a disporsi a un nuovo ascolto, quello diretto - ma mediato dal contesto performativo - dei suoni del mondo.

Benveniste (1970), nella sua teoria dell'enunciazione, formula una precisa definizione della temporalità, distinguendo un tempo della storia e un tempo del discorso, che si colloca sempre nel presente. Più specificamente, esiste un tempo fisico, infinito e uniforme, lineare e irreversibile. È il tempo della vita. Esiste poi un tempo cronico, quello scandito dagli strumenti di misurazione, un tempo oggettivato e socializzato che ordina gli eventi e permette di distinguerne la disposizione lungo l'asse del tempo fisico. Il tempo dell'orologio che l'esecutore cageano deve sempre avere con sé. Esiste, infine, un tempo linguistico, in cui si realizza l'enunciazione.

Le présent est proprement la source du temps. Il est cette présence aumonde que l'acte d'énonciation rend seul possible, car, qu'on veuille bien y réfléchir, l'homme ne dispose d'aucun moyen de vivre le «maintenant» et de le faire actuel que de le réaliser par l'insertion du discours dans le monde. (Ibid.)

L'inserzione è dunque il momento in cui l'enunciato si realizza nel tempo fisico, attraverso la creazione di uno spazio temporale. Nel flusso continuo e indistinto del paesaggio sonoro, 4'33" funziona come un buco nella rete montaliana. Cage apre un varco nell'ascolto abbacinato e stordito dei suoni d'ambiente e dischiude un frangente di vitalità e consapevolezza; è autore del gesto e affida alle cose del mondo il compito di stupirci. "Una cesura protratta nel tempo", aveva detto Schafer in riferimento a 4'33" (1977).

Ogni azione che intende isolare, riproporre, ricomporre il paesaggio sonoro produce un significato nuovo; è l'enunciatore, per quanto taciturno possa essere, che realizza il significato della lingua. 


\section{Il SUONO COMUNICATO}

A coniare il termine soundscape non fu Schaefer. Il neologismo è storicamente attribuito all'urbanista Michael Southworth che lo introdusse nella sua tesi di laurea, ma fu in realtà fu Buckminster Fuller, in un intervento del 1964 dal titolo Epigenetic Landscape a parlare di soundscape per evidenziare la reciprocità evolutiva all'interno della relazione suono/ uomo/linguaggio/ambiente: "when man invented words and music he altered the soundscape and the soundscape altered man. The epigenetic evolution interacting progressively between humanity and his soundscape has been profound» (1966).

La musica, come la parola, altera il paesaggio sonoro, che, in un moto reciproco, altera l'uomo. Nel testo Acoustic communication del 1984, Barry Truax, membro originario del World Soundscape Project si riferisce al paradigma di "comunicazione acustica" come modalità generale per descrivere qualsiasi situazione acustica che coinvolga un ascoltatore. Suono, ascoltatore (che è anche, a sua volta, produttore di suoni) e spazio si trovano in una interrelazione continua, all'interno di un rapporto che coinvolge i singoli, ma anche le comunità:

acoustic communication attempts to understand the interlocking behavior of sound, the listener and the environment as a system of relationships, not as isolated entities. The listener is also a soundmaker, and even the sound of one's own voice comes back to the ear colored by the environment. With sound, everything interacts with everything else. (1984)

In un capitolo dedicato ai tre principali sistemi di comunicazione acustica - parola/musica/ambiente dei suoni - Truax pone queste categorie, dai confini molto labili, lungo un continuum. Una delle variabili attraverso cui è possibile leggere questo continuum è quella del senso. Muovendoci dall'ambiente dei suoni alla parola attraverseremo codici la cui densità e specificità semantica è sempre maggiore. L'ambiente dei suoni è per Truax di per sé suono organizzato (perché generato anche da artefatti umani e dall'uomo stesso), ma risulta più difficilmente e meno frequentemente significativo. La parola è il sistema più denso e specifico.

Il rapido percorso tracciato in questo articolo ci mostra che la musica, lo stadio di mezzo, che si è appropriato gradualmente dell'ambiente dei suoni, ne imita le forme e le influenza. Le traduce, attraverso il filtro della percezione soggettiva, in senso e ne rende più facilmente possi- 
bile una percezione estetica, aumentandone la pregnanza semantica. 亡̀ per questo un linguaggio inalienabile e fondamentale per perseguire le istanze del paesaggio sonoro: per dire quasi la stessa cosa, stimolando il desiderio dell'ascoltatore, generando un valore immaginativo, brivido di velluto nelle sue orecchie.

\section{RIFERIMENTI BIBLIOGRAFICI}

Benveniste, E. 1970. "L'appareil formel de l'énonciation". Langages, 17.

Bonnet, F. 2016. The order of sounds. A sonorous archipelago. Windsor Quarry, Falmouth (UK): Urbanomic.

Chion, M. 2004. L'arte dei suoni fissati. Roma: Edizioni Interculturali.

Fuller, B. 1966. "The music of the New Life". Music Educators Journal, 52(6).

Grubbs, D. 2014. I dischi rovinano il panorama. Roma: Arcana.

Husserl, E. 1965. Idee per una fenomenologia pura e per una filosofia fenomenologica. Torino: Einaudi.

Lévinas, E. 1998. Altrimenti che essere o al di là dell'essenza. Milano: Jaca Book.

Kelman, A. 2010. "Rethinking the soundscape". Senses and Society, 5(2).

Ponzio, A. ed. 2006. Alle radici del senso con Roland Barthes. Roma: Meltemi.

Schaeffer, P. 1996. Traité des object musicaux. Paris: Seuil.

Schafer, R.M. 1985. Il paesaggio sonoro [1977]. Milano: Ricordi LIM.

Serra, C. 2005. La rappresentazione fra paesaggio sonoro e spazio musicale. Milano: Unicopli.

Truax, B. 1984. Acoustic communication. Westpoint: Ablex Publishing. 
